RESEARCH HIGHLIGHTS

\title{
Missing the beat
}

Recently, phospholipase C $\gamma 1$ (PLCG1) was shown to be a key regulator of arterial vasculogenesis in vertebrates. Now, using zebrafish, Wolfgang Rottbauer, Steffen Just and colleagues show that this enzyme also has an earlier and more fundamental role in regulating the contractility of the embryonic heart.

The authors identifed dead beat in a forward genetic screen for lethal mutations that perturb cardiac function. As well as showing progressive, ventricle-specific reduction of cardiac contractility, the mutant embryos also have defects in embryonic vasculogenesis, and lack a lumenized dorsal aorta and the posterior cardinal vein.

Rottbauer et al. mapped the dead beat mutant to the zebrafish plcg1 gene, and found that it had a premature stop codon induced by a G-to-T transition. Two experiments nailed down plcg1 as the gene involved in the dead beat phenotype. An antisense morpholino to the translational start site or the splice donor site of exon 13, which results in abnormal splicing products, mimicked the effects of the dead beat mutation: mutants had lower ventricular contractility and had no blood circulating in lumenized vessels. In addition, injecting wild-type plcg1 mRNA into dead beat mutant embryos completely rescued both the vascular and heart phenotypes in $70 \%$ of the cases. The expression pattern of plcg1 is consistent with its biological function: its RNA is ubiq- uitously present in zebrafish embryos and is more pronounced in the brain, vasculature and heart.

Plcg1 fuctions downstream of vascular endothelial growth factor (Vegf) signalling in many developmental processes and the authors found that Vegf is essential for zebrafish cardiac contractility. They also found that the effect is mediated by Vegf receptor 1 (Flt1). But does the signalling cascade through VEGF-FLT1-PLCG1 also affect cardiac myocyte contractility in mammals? By using specific pharmacological inhibitors for each of these molecules in rat ventricular cardiomyocytes, they found that PLCG1 signalling controls mammalian cardiomyocyte contractility by modulating calcium cycling.

The authors conclude that heart muscle uses this cascade to control

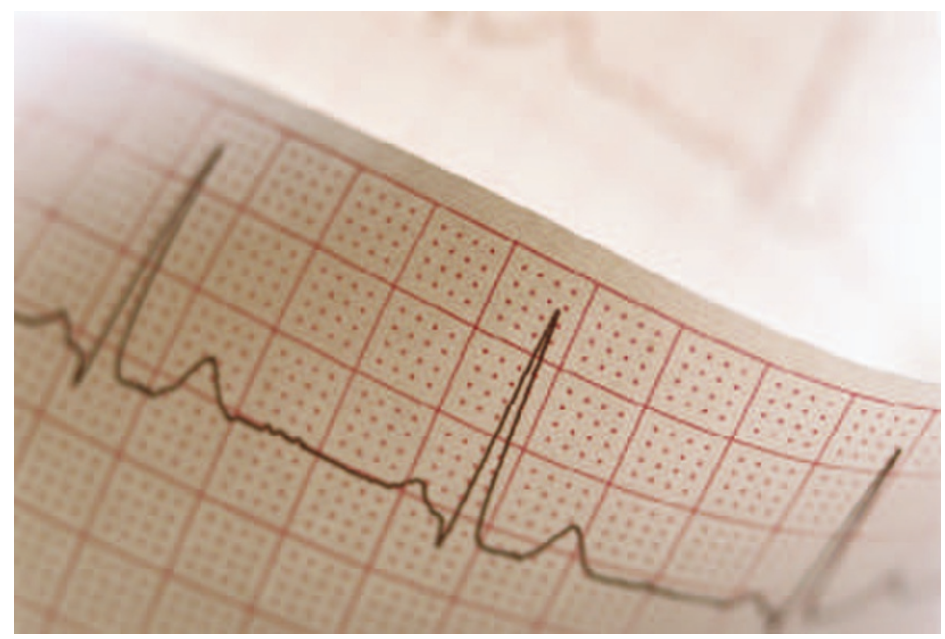

the strength of the heart beat and speculate that the VEGF-PLCG1 pathway might contribute to the normal and pathological regulation of cardiac contractility. Heart failure is a very common disease in humans, and, as no agents are currently available to safely enhance cardiac contractility, it would be of interest to examine whether the VEGF-PLCG1 pathway might offer ment.

\section{(2) References and links} ORIGINAL RESEARCH PAPER Rottbauer, W. et al.

VEGF-PLC 1 pathway controls cardiac contractility in the embryonic heart. Genes Dev. 1 WEB SITES

Mark Fishman's web page: http://www. massgeneral.org/cvrc/crvc/fishman.html Wolfgang Rottbauer's web page: http://www. klinikum.uni-heidelberg.de/index.php?id=4580 new opportunities for such treatJuly 2005 (doi:10.1101/gad.1319405) 